\title{
Atlas of Cancer Signaling Network: A resource of multi-scale biological maps to study disease mechanisms
}

L Cristobal Monraz Gomez ${ }^{1}$, Maria Kondratova ${ }^{1}$, Nicolas Sompairac ${ }^{1}$, Christine Lonjou ${ }^{1}$, Jean-Marie Ravel ${ }^{2}$, Emmanuel Barillot $^{1}$, Andrei Zinovyev ${ }^{1}$, Inna Kuperstein ${ }^{1}$

${ }^{1}$ Institut Curie, PSL Research University - INSERM U900 - MINES ParisTech, PSL Research University

${ }^{2}$ Laboratoire de génétique, Centre Régional Hospitalier Universitaire de Nancy, Vandœuvre-lès-Nancy, INSERM UMR 954, Université de Lorraine, Vandœuvre-lès-Nancy

26 Rue d'Ulm, 75248 Paris cedex 05, France

inna.kuperstein@curie.fr Tel.: +33 (0) 156246987; Fax: +33 (0) 156246911

\section{Keywords}

Biocuration, Cancer, Data visualization, Enrichment analysis, Knowledge formalization, Modelling, Molecular network, Network navigation, Signaling network map, Signatures, Structural analysis, Systems biology, Systems medicine

\section{Abstract}

ACSN (https://acsn.curie.fr) is a web-based resource of multi-scale biological maps depicting molecular processes in cancer cell and tumor microenvironment. The core of the Atlas is a set of interconnected cancer-related signaling and metabolic network maps. Molecular mechanisms are depicted on the maps at the level of biochemical interactions, forming a large seamless network. The Atlas is a "geographic-like" interactive "world map" of molecular interactions leading the hallmarks of cancer as described by Hanahan and Weinberg. The Atlas is created using the systems biology standards and therefore is amenable for computational analysis. The maps of ACSN are organized in a hierarchical manner and decomposed into functional modules with meaningful network layout. Navigation of the ACSN is intuitive thanks to the Google Maps-like features in the NaviCell web platform. Particularly, the exploration of the Atlas is simplified due to the semantic zooming feature, allowing the user to visualize the seamless Atlas and individual maps from the collection at different levels of details. The resource includes tools for visualization and analysis of molecular data in the context of signaling network maps. In this chapter we present how a disease-specific resource such as the Atlas of Cancer Signaling Network (ACSN) can be useful to study and interpret molecular perturbations in cancer, among others, explaining drug resistance, suggesting intervention points, finding phenotype shifts through disease progression, explaining susceptibility to a particular type of cancer and studying disease comorbidities.

\section{Nomenclature}

ACSN: Atlas of Cancer Signaling Network

CAF: Cancer associated fibroblast

EMT: Epithelial to Mesenchymal Transition

GSEA: Gene Set Enrichment Analysis

MCS: Minimal cut sets

PARP: Poly-ADP-ribose polymerase

SL: Synthetic Lethal

TNBC: Triple Negative Breast Cancer 


\section{Introduction}

Carcinogenesis represents the aberrant functioning of a complex network of molecular interactions and processes, such as the cell cycle, regulated cell death, DNA repair and replication, cell motility and adhesion, cell survival mechanisms, immune processes, angiogenesis, tumor microenvironment, and many others. These processes are collectively or sequentially involved in tumor formation and modified as the tumor evolves (Hanahan and Weinberg, 2011).

The scientific literature often suggests that, in pathological situations, the normal cell signaling network is altered by deregulated coordination between pathways or disruption of existing molecular pathways, rather than by creating completely new signaling pathways. The most common abnormalities in pathological situations are perturbations at the gene expression level, protein abundance or protein posttranslational modifications, irregular 'firing' or silencing of particular signals, wrong subcellular localization of particular molecules and so on. Such quantitative rather than qualitative network changes, compared with the normal cell signaling, could be studied in the context of comprehensive signaling networks by analyzing experimental data obtained from tumor samples, patient-derived xenografts, cancer-related cell lines or animal models. This approach helps to understand the interplay between molecular mechanisms in cancer and to decipher how gene and protein interactions govern the hallmarks of cancer (Hanahan and Weinberg, 2011) in specific settings.

Despite the existence of a large variety of pathway databases and resources (Chowdhury and Sarkar, 2015), only few of them are cancer-specific, and none of these resources depict the processes with enough granularity. In addition, pathway browsing interfaces are becoming more important for cancer researchers and clinicians however, further improvements are required.

Therefore, we constructed a resource, the Atlas of Cancer Signaling Network (ACSN, http://acsn.curie.fr), a web-based multi-scale resource of signaling network maps depicting molecular processes in the cancer cell and tumor microenvironment. These signaling network maps are organized by themes, each describing a major signaling mechanism, and are bound together in the form of a global map that we call "Atlas". The construction and update of ACSN involves manual curation and mining of the literature in cellular and molecular biology, together with the contribution of experts in those fields. The cell signaling mechanisms are depicted using the CellDesigner tool (Kitano et al., 2005) at the level of biochemical interactions, assembling a large seamless network. The ACSN is applicable for data visualization, pathway definitions, and structural analyses, suitable for systems medicine (Kuperstein et al., 2015). The cell signaling mechanisms are depicted in great detail, creating together a map of molecular interactions, presented as a global 'geographic-like' representation. ACSN has a hierarchical structure; each map is divided into functional modules, corresponding mainly to canonical signaling pathways. Currently the Atlas is being extended with additional maps depicting other important processes in the cancer cell as well as the components of the tumor microenvironment. An additional level of complexity will be added to the Atlas in the near future, representing different types of cells surrounding the tumor, and their interplay, to enable modelling of complex phenotypes.

The navigation and website availability of ACSN is possible thanks to NaviCell web platform. NaviCell (https://navicell.curie.fr) is a web-based environment empowered by Google Maps (Kuperstein et al., 2013). The maps generated with CellDesigner can be integrated in NaviCell, and the navigation features such as scrolling, zooming, markers, callout windows and zoom bar, are adopted from the Google Maps interface. The NaviCell web service can be used to import and visualize expression data (for molecular entities such as mRNAs, proteins, and miRNAs), gene copy-number data, mutation data and simple gene lists, such as small interfering RNA (siRNA)-based screening hit list. In a more elaborated situation, a weighted gene list can be visualized such as the distribution of mutation frequencies observed in multiple cancers. NaviCom (https://navicom.curie.fr) is a platform to generate interactive network based molecular portraits using high-throughput datasets. Additionally, NaviCom connects with the cBioPortal database 
(www.cbioportal.org) (Cerami et al., 2014) and NaviCell web service, and it allows to display various high-throughput data types simultaneously on the network maps in a user-friendly way (Dorel et al., 2017).

ACSN is a unique resource of cancer signaling knowledge, with a vast amount of information embedded and organized. Together with NaviCell, it is optimized for integration and visualization of cancer molecular profiles generated by highthroughput technologies, drug screening data or synthetic interactions studies. The integration and analysis of these data in the context of ACSN may help to better understand the biological relevance of results, guiding scientific hypotheses and suggesting potential therapeutic interventions for cancer patients. Data visualization, network and pathway definitions, structural analysis and large genomic studies are just few of the available applications of ACSN; however, we will use some examples from those fields to demonstrate the ACSN's reach.

This chapter is organized into four sections, dedicated to applications of ACSN in systems medicine. We present here how a disease-specific resource like ACSN can help to study and interpret molecular perturbations in cancer, among others, explaining drug resistance, suggesting intervention points, finding phenotype shifts through disease progression, explaining susceptibility to a particular type of cancer and studying disease comorbidities.

\section{ACSN applications for data omics analysis and visualization}

\section{Explaining the synergistic effect of combined treatments in breast cancer}

Synthetic lethality (SL) provides a conceptual frame to develop cancer-specific drugs. This classical paradigm describes $S L$ interactions as a phenomenon where combinations of two gene deletions significantly compromise cell viability, whereas the deletion of one of those genes does not. Targeting the SL partner allows the selective killing of the tumor cells. Due to the complexity of signaling mechanisms occurring in cancer simultaneously, the SL pair paradigm should be extended to the SL sets or combinations paradigm (Garg et al., 2013), (Huang et al., 2014).

DNA repair inhibitors are holding promises to improve cancer therapy. For example, PARP inhibitors, which act as SL with BRCA deficiency, appear less efficient in patients with active Homologous Recombination (HR) repair mechanisms (Lord et al., 2015). During treatment, some tumors escape the elimination through compensatory mutations that restore the HR activity or stimulate the activity of alternative repair pathways. Therefore, a new class of DNA repair pathways inhibitors (Dbait or AsiDNA, a Dbait derivative) has been recently developed, consisting of 32 bp deoxyribonucleotides DNA double helix that mimics double-strand breaks (DSB). It acts as an agonist of DNA damage signaling, thereby inhibiting DNA repair enzyme recruitment at the damage site (Quanz et al., 2009). However, studies on the effects of Dbait on multiple types of cancer cell lines show occurrences of resistance in a cancer typeindependent manner.

Depending on the genetic background, different breast cancer tumors vary in their sensitivity to DNA repair inhibitors, as PARP inhibitors and AsiDNA. Triple negative breast cancer (TNBC) cell lines were studied for their sensitivity to AsiDNA, the derivative of Dbait DNA repair inhibitor, and Olaparib, the PARP inhibitor. Different TNBC cell lines show a wide distribution of response/resistance to these drugs, despite the fact that these cell lines are related to the same disease type. Integrative analyses of omics data (mRNA expression, copy number variations and mutational profiles) from these cell lines were performed, retrieving non-overlapping unique gene sets robustly correlated with resistance to each one of the drugs. Enrichment analysis of the omics data in the context of ACSN maps highlighted dysregulated functional modules across ACSN, associated with resistance to each one of the drugs allowing establishing drug resistance network-based molecular portraits. This analysis confirmed that different specific defects in the DNA repair 
machinery are associated to AsiDNA (Figure 1A) or Olaparib (Figure 1B) resistance. Importantly, it showed involvement of different compensatory DNA repair mechanisms in cell lines resistant to AsiDNA when compared with cell lines resistant to Olaparib (Figure 1D), suggesting a rationale to combine these two drugs. The synergistic therapeutic effect of the combined treatment with AsiDNA and PARP inhibitors in TNBC has been experimentally confirmed, while sparing the healthy tissue (Figure 1C) (Jdey et al., 2017).

\section{Figure 1}
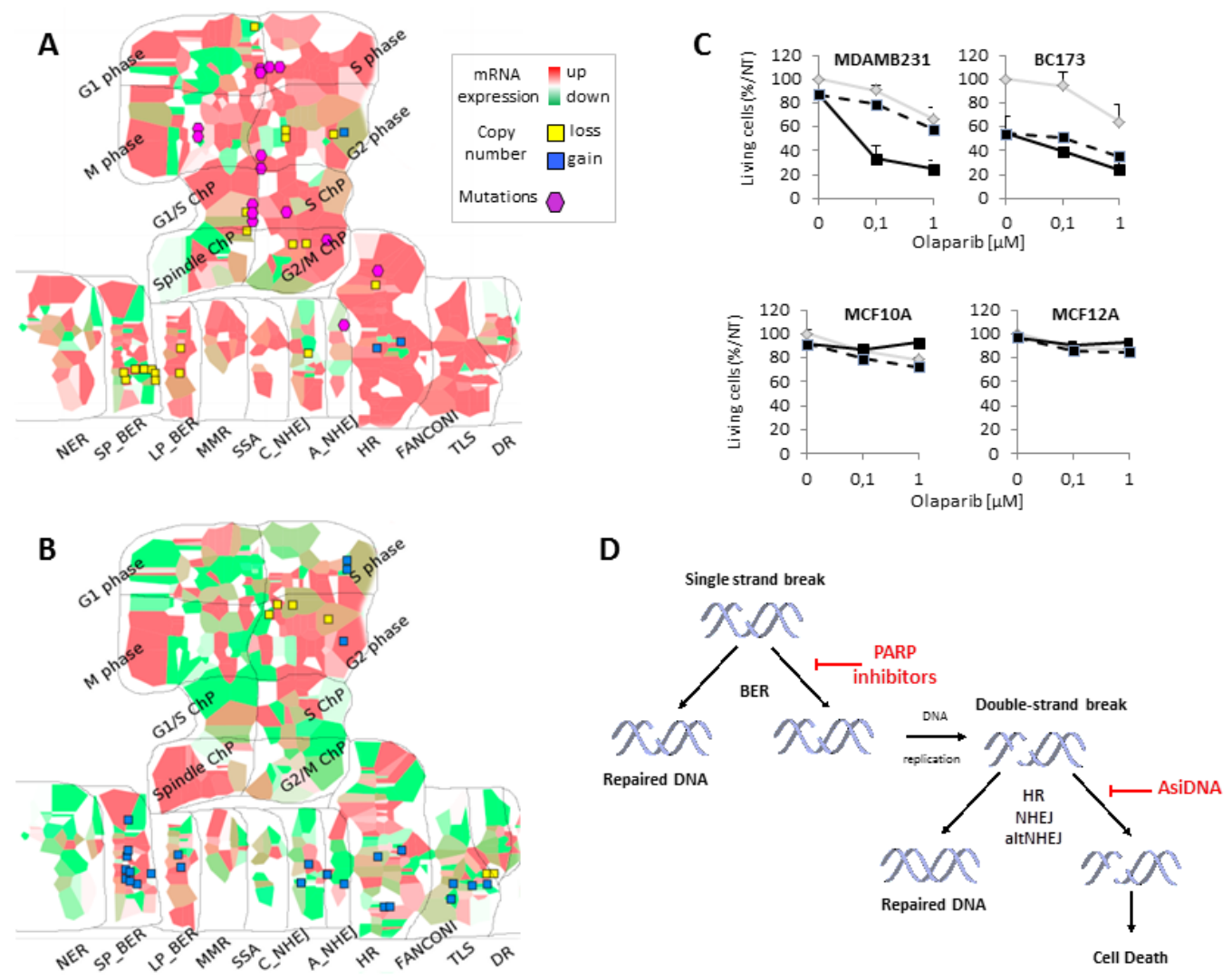

Figure 1. Restoring sensitivity of TNBC cell lines to DNA repair inhibitors. Visualization of expression, copy number and mutation profiles of TNBC cell lines resistant to (A) AsiDNA or (B) Olaparib on the DNA repair map. (C) Cell survival rates to combination of AsiDNA and Olaparib, with AsiDNA 1 (black line), without AsiDNA (grey line); dashed lines indicate calculated cell survival for additive effect of both drugs. (D) Representation of inhibitory mechanisms of AsiDNA and Olaparib. Base excision repair (BER), HR, NHEJ, Alt-NHEJ Red-up-regulated and green to down-regulated functional modules. (Adapted from Jdey et al., 2017)

\section{Cell type-specific signaling network maps help to reveal signatures of cell heterogeneity and polarization in tumor microenvironment}

The molecular complexity of tumor microenvironment (TME) creates a bottleneck in interpretation of cancer omics data. To address this challenge, we demonstrate that systematic graphical representation of cell type-specific molecular mechanisms governing polarization of different TME components is an important step in data interpretation. To describe the balance between components of the TME and to analyze impact of non-immune cells as cancer associated fibroblasts (CAF), information on related molecular mechanisms was systematically collected and represented in a form of a comprehensive network map. The modular map covers the main functions of CAFs in cancer as interactions with extracellular matrix components, signaling coordinating involvement of CAFs in tumor growth and interactions of CAFs with immune system in TME. The CAF network map contains eleven functional modules, showing mechanisms associated with pro-tumoral CAF activity. 
The analysis and interpretation of gene expression patterns from breast cancer CAFs samples in the context of the network map helped to characterize different CAF subsets with distinct molecular properties (Costa et al., 2018). The enrichment analysis of transcriptome data using the gene sets from functional modules of CAF map highlighted that CAF-S1 subset exhibits a high expression of immune signatures, including cytokines production and modulation of regulatory T lymphocytes (Tregs), promoting an immunosuppressive microenvironment. Whereas, CAF-S4 subset exhibits matrix regulation and motility mechanisms, indicating that the CAF-S4 subset most probably modulates properties of extracellular matrix and facilitates tumor invasion. These results where visualized on the maps by using the "map staining" technique that displays the loaded data at the background of the map as described in Bonnet et al., 2015). These stained maps serve as indicators of the processes that might be more or less active by the coloring in the different zones of the map and help to interpret enrichment results from the biological point of view (Figure 2). The application of this systems biology resource for identification of CAFs subset in breast cancer has a broader context for studying different components of TME.

\section{Figure 2}
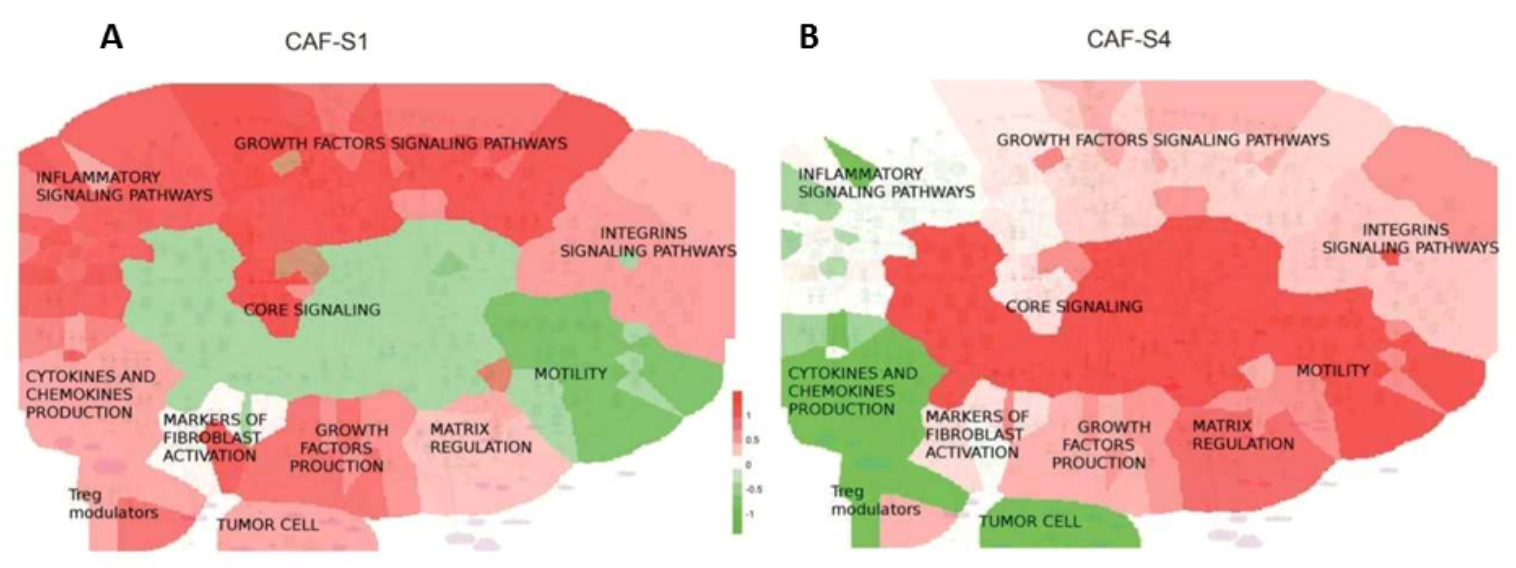

Figure 2. Visualization of transcriptome profiles for (A) CAF-S1 and (B) CAF-S4 in the context of cell type-specific CAF map. Red highly expressed and green lowly expressed functional modules. Boxes represent differentially expressed modules related to interactions with the immune system.

\section{Regulated Cell Death Map in disease comorbidity studies}

Based on experimental data retrieved from literature, an integrated signaling network of a Regulated Cell Death (RCD) map has been constructed. The RCD map was applied to explore the differences in cell death regulation between Alzheimer's disease (AD) and lung cancer. $A D$ is a disease where neurodegeneration acts as the main trigger of cognitive decline and other symptoms (Sperling et al., 2011). Nevertheless, the precise molecular processes resulting in neuron death remain uncertain. In order to explore if the regulated cell death mechanisms play a role in $A D$, we analyzed gene expression data from different studies. Additionally, in several epidemiological and other studies, it has been suggested that lung cancer has an inverse comorbidity (lower than expected co-occurrence (Tabarés-Seisdedos and Baudot, 2016)) with AD (Musicco et al., 2013; Ou et al., 2012; Sánchez-Valle et al., 2017). Since cancer has been described as a disease where cell survival is abnormally maintained, evading programmed cell death, the comparative study of three lung cancer and AD datasets was performed. 
Enrichment analysis of RCD map functional modules using gene expression data was performed using the ROMA algorithm (Martignetti et al., 2016); deregulated molecular functions as well as the main players were compared. In the AD data, the glucose metabolism, mitochondrial genes, as well as the oxidative phosphorylation and TCA cycle modules were significantly over-dispersed in the three studies, whereas in the lung cancer data ER stress was the only over-dispersed module across the three studies. Nevertheless, we found a downward trend in modules related to mitochondrial function in AD cases compared to lung cancer. Interestingly, the oxidative phosphorylation and TCA cycle module exhibits a trend to score higher in lung cancer than in AD (Figure 3).

Moreover, when we used the map-staining technique to visualize the aforementioned values, we identified additional differences in the regulation of RCD-related processes between the two diseases. Namely, the pyroptosis module appeared to be more active in $A D$ vs. lung cancer. Experimental evidence has shown the Pyroptosis in $A D$ to be a response to the NLRP1 inflammasome (Kaushal et al., 2015) or NLRP3 inflammasome (Schmid-Burgk et al., 2016). However, there are no studies correlating $A D$ and pyroptosis directly in humans.

In addition, the visualization of data in the context of the RCD map highlighted a persisting activation of metabolicrelated modules, as well as ER stress processes in lung cancer in contrast to AD (Figure 3C-D). The literature suggests that endoplasmic reticulum stress is as an adaptive response that could lead to either tumor growth or apoptosis in tumors (Avril et al., 2017; Giampietri et al., 2015). In addition, this response has also been related to chemotherapy resistance (Salaroglio et al., 2017). This study allowed identifying that metabolism-related modules are less active in $A D$ comparing to lung cancer. We concluded that the inverse comorbidity between these diseases implicates rather metabolic pathways but further research is needed. 


\section{Figure 3}

A

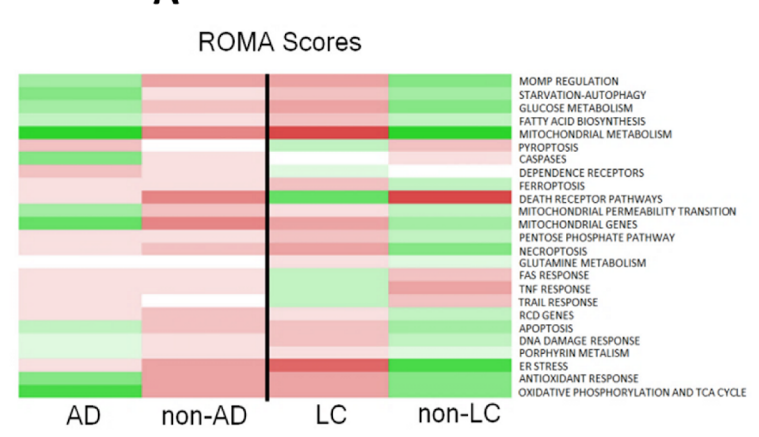

B

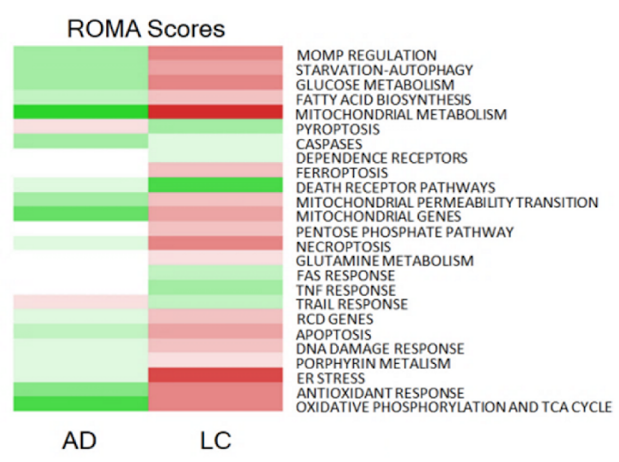

C

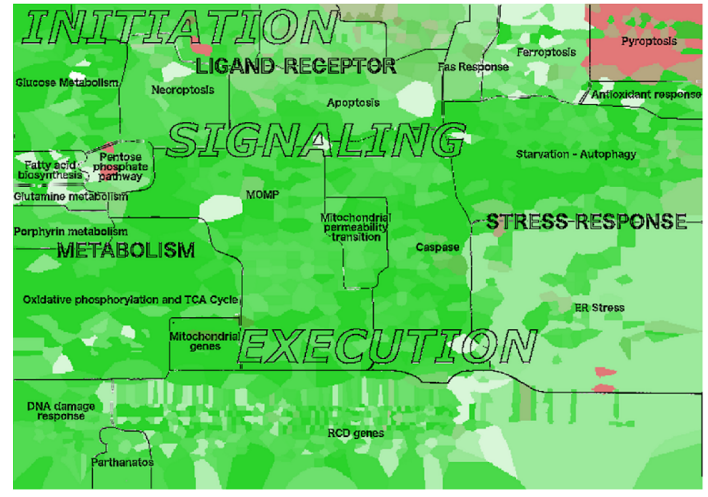

D

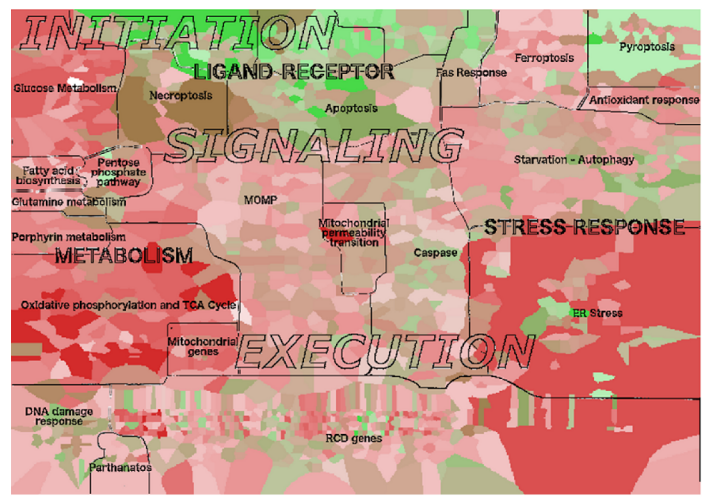

Figure 3. Comparison of transcriptomic profiles between Alzheimer's disease and lung cancer using Regulated Cell Death signaling map. A) Heatmap representing the average enrichment ROMA scores across the data sets used per group (AD: Alzheimer's disease, non-AD: non Alzheimer's disease, LC: lung cancer, non LC: non lung cancer). B) Heatmap representing the disease average enrichment ROMA after subtracting the non-diseased scores. Visualization of the enrichment ROMA scores, from figure $B$, on the RCD map, for (C) AD and (D) lung cancer datasets. Red highly active and green lowly active functional modules.

\section{ACSN applications for defining rewired pathways in cancer}

\section{ACSN as a source of informative signatures of cancer}

ACSN can be used in various computational biology studies as a source of pathway definitions in terms of the sets of genes composing them or the structure of the connections between pathway members. ACSN and other pathway databases have been assessed regarding the value of pathway gene sets for downstream analyses, for example in functional enrichment studies (Cantini et al., 2018). A formalism of informative signatures, using an unsupervised approach to prioritize gene sets capable of defining a natural ranking of samples, has been developed for this purpose (Cantini et al., 2018), and pathway definitions, both from knowledge-based databases like ACSN and from data-driven approaches like MSigDB (C4 computational gene sets, (Liberzon et al., 2015) were assessed for their chance to obtain significant enrichment scores in transcriptomic studies in cancer research.

With this aim, a large corpus of data composed of 8991 transcriptomically profiled tumor samples for 32 cancer types from the Cancer Genome Atlas (TCGA, https://portal.gdc.cancer.gov/) was analyzed with 12096 definitions of pathways from various pathway databases, including ACSN. Then the pathway definitions were ranked in terms of the 
variance explained by the genes in the pathway, called eigen-genes, (overdispersion) and the spectral gap between the first and the second pathway eigene values (overcoordination). (Martignetti et al., 2016). In a series of computational experiments, it was demonstrated that ACSN pathways have a higher chance to obtain significant enrichment scores when comparing typical groups of tumoral transcriptomes i.e., in normal vs cancer transcriptome comparisons, or tumors of different stages. It was concluded that ACSN pathway definitions are relatively more informative for cancer studies than the definitions coming from other knowledge-based pathway databases such as REACTOME or KEGG (Figure 4A).

\section{ACSN as a source of network for finding correlation between gene profiles}

Definitions of pathways from ACSN have been used also for demonstrating the utility of several computational methods. The Cytoscape plugin DeDaL (Data-Driven network Layout) provides an algorithm for constructing datadriven 2D network layouts, which simultaneously reflects the structure of the network of interactions and the proximity between genes in the metrics defined by the omics data. This approach is useful for example, for finding correlations between gene profiles in a cohort of patients (Czerwinska et al., 2015). An example of such data-driven network layout for one of the pathways existing in ACSN, the Fanconi module of DNA repair map, is shown in Figure 4B.

Figure 4

A

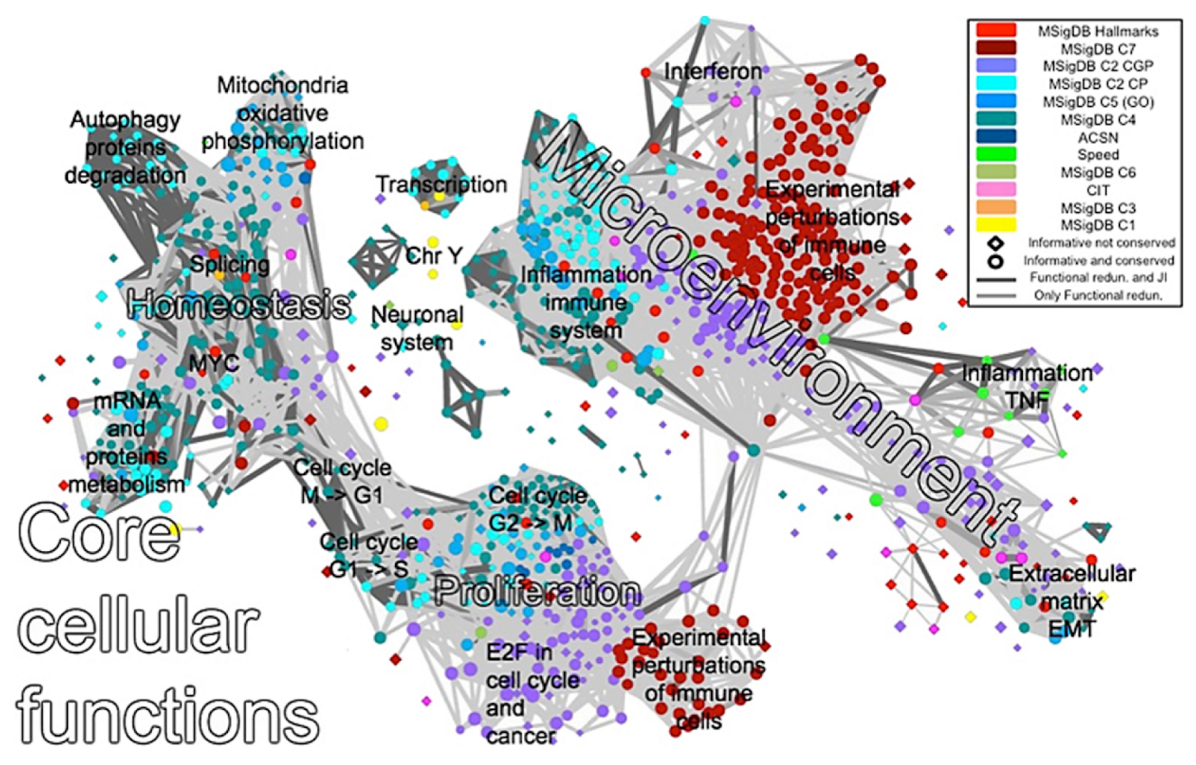

B

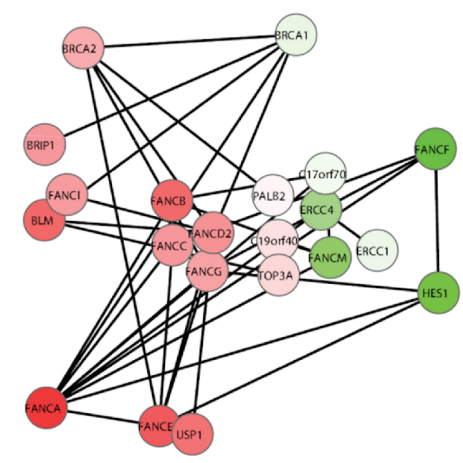

C

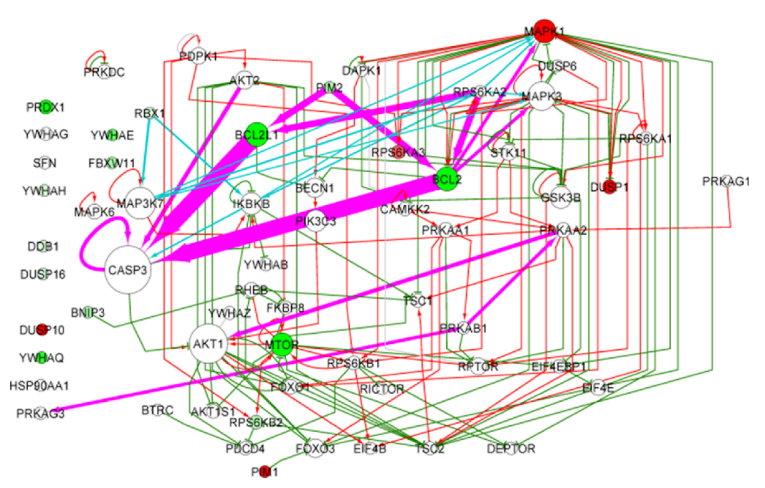

Figure 4. ACSN applications for defining rewired pathways in cancer. A) ACSN as a source of informative cancer signatures in the InfoSigMap. B and C) Examples of using ACSN in validating computational methods. B) Data-driven network layout of Fanconi pathway structure taken from ACSN DNA repair map, produced by DeDaL Cytoscape plugin (Czerwinska et al., 2015). Colors here visualize the t-test value for comparison the gene expression profile in basal vs non-basal breast cancers (green for downregulated 
in basal subtype and red for upregulated). C) Application of the reduced Google matrix method for the analysis of AKT-mTOR pathway from ACSN (Lages et al., 2018). Here the color of the edges shows either the sign of the regulation (green for inhibition and red for activation) or inferred hidden interactions between pathway members (purple) or hidden interaction emerging in cancer cells due to the changes in the underlying transcriptional network (light blue). The color of the node shows the sign of the change of the global node PageRank in cancer cell vs normal cell.

\section{Rewired pathways in leukemia cell lines}

The Google matrix approach, based on a random walk method, is a well-established tool to analyze complex directed networks. In systems biology, this approach can be applied to evaluate the features of signal propagation through a pathway by considering a stochastic Markov chain with uniform non-zero restart probability along oriented edges of the graph representing the global biological network. Following this framework, the inference of hidden causal relations between the members of a signaling pathway or a functionally related group of genes, is possible.

A definition of the AKT-mTOR pathway from ACSN was used to validate the reduced Google matrix approach, aimed at inferring hidden indirect connections between pathway members through the global interaction network (Lages et al., 2018). By comparing normal lymphocytes with a leukemia cell line, it was demonstrated that certain parts of the AKT-mTOR pathways have been indirectly rewired due to the changes in the underlying global transcriptional regulatory network (Figure 4 C).

\section{ACSN network structure analysis applications to reveal key regulators of biological processes}

\section{Finding metastasis inducers in colon cancer through network analysis}

Invasion and metastasis, in particular in colon cancer, have been extensively studied in experimental models. The mechanisms that trigger the process are still largely unknown, and the available mouse models of colon cancer are far from being satisfactory (Hung et al., 2010; Trobridge et al., 2009). To generate an effective experimental mouse model of invasive colon cancer, it is mandatory to understand the key players that lead to invasion. Since Epithelial-toMesenchymal Transition (EMT), a type of differentiation process where epithelial cells lose their polarity and acquire a motile mesenchymal phenotype, is assumed to be an early event in the multi-step invasion process (Nieto, 2011), understanding on/off mechanisms of EMT represents the starting point.

To identify the interplay between signaling pathways regulating EMT, a signaling network was created. This signaling map is integrated into the EMT and cell motility comprehensive map of ACSN (https://acsn.curie.fr/navicell/maps/emt senescence/master/index.html). Structural analysis and simplification of the EMT network highlighted the EMT network organization principles (Chanrion et al., 2014), which agree with current EMT understanding (Fre et al., 2009; Knouf et al., 2012; Moes et al., 2012; Siemens et al., 2011). Based on those features, network complexity reduction was performed using the Cytoscape plugin BiNoM (Zinovyev et al., 2008). The reduced network contained the core regulatory cascades of EMT, apoptosis and proliferation that were preserved through all reduction levels (Bonnet et al., 2013). This reduced network has been used for comparison between the wild type and all the possible single and double mutant combinations that could promote EMT. The computational analysis of the signaling network led to the prediction that the simultaneous activation of Notch Intracellular Domain (NICD) and loss of p53 can promote an EMT phenotype. Furthermore, EMT inducers may activate the Wnt pathway, 
possibly producing a positive feedback loop that will amplify Notch activation and maintain an EMT-like program (Figure 5A-C). To validate this hypothesis, a transgenic mouse model was generated, expressing a constitutively active Notch1 receptor in a p53-deleted background, specifically in the digestive epithelium. Importantly, green fluorescent protein (GFP) expression linked to the Notch1 receptor activation allows lineage tracing of epithelial tumor cells during cancer progression and invasion (Figure 5D). These mice developed digestive tumors with dissemination of EMT-like epithelial malignant cells to the lymph nodes, liver and peritoneum, and they generated distant metastases (Figure $5 \mathrm{E}$ ). Exploration of early EMT program inducers in invasive human colon cancer samples confirmed that EMT markers are associated with modulation of Notch and p53 gene expression in a similar manner as in the mouse model (Figure $2 \mathrm{~F}$ ), supporting a synergy between these genes to induce EMT (Chanrion et al., 2014). The prediction of synthetic interaction between Notch (NICD) and p53 demonstrated that there are alternative ways to achieve conditions permissive of EMT, beyond those already described in literature. This result was not obvious from the previous data and partially contradicts the commonly accepted dogma in the colon cancer field. Gathering together cell signaling mechanisms may uncover unexpected interactions and lead to the finding of regulatory mechanisms of cell phenotypes that might significantly affect our understanding of molecular processes implicated in cancer, hence changing therapeutic approaches. Finally, the new EMT mice are a relevant model mimicking the invasive human colon cancer and a system for therapeutic drug discovery (Inna Kuperstein et al., 2015b).

Figure 5

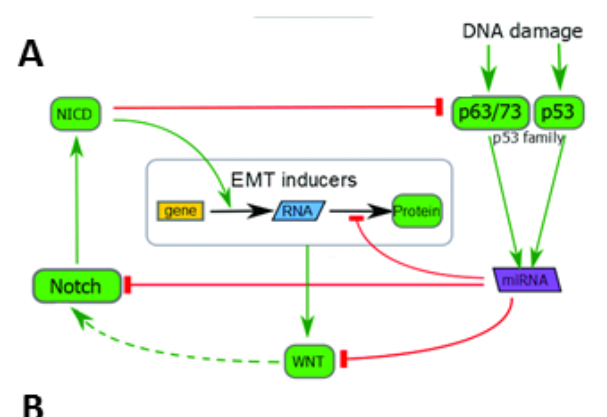

B

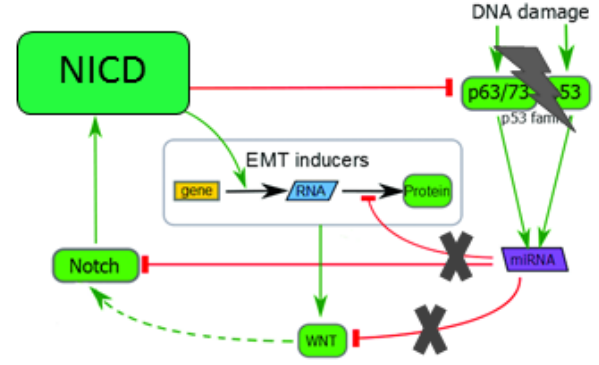

E

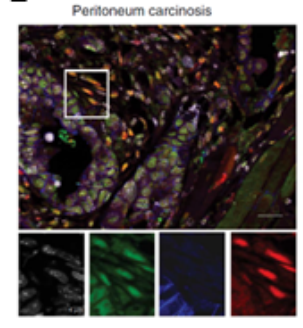

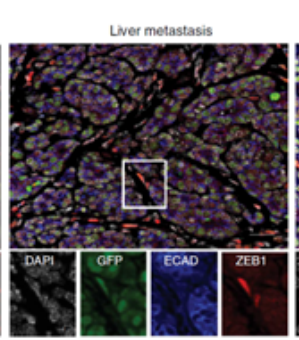

C

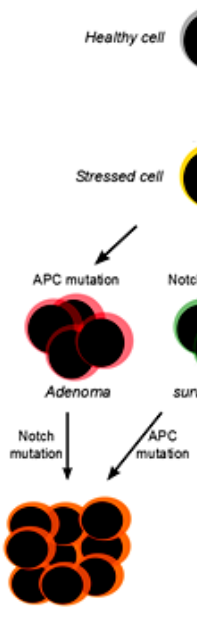

Proliferation

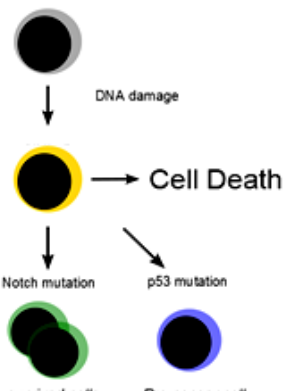

survived cells Pre-cancer cell

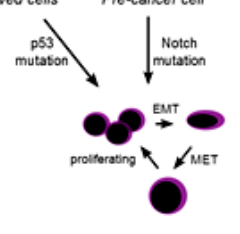

Metastasis
D
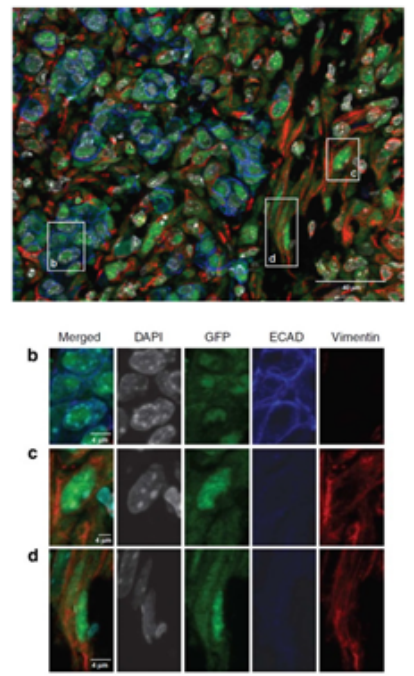

$\mathbf{F}$
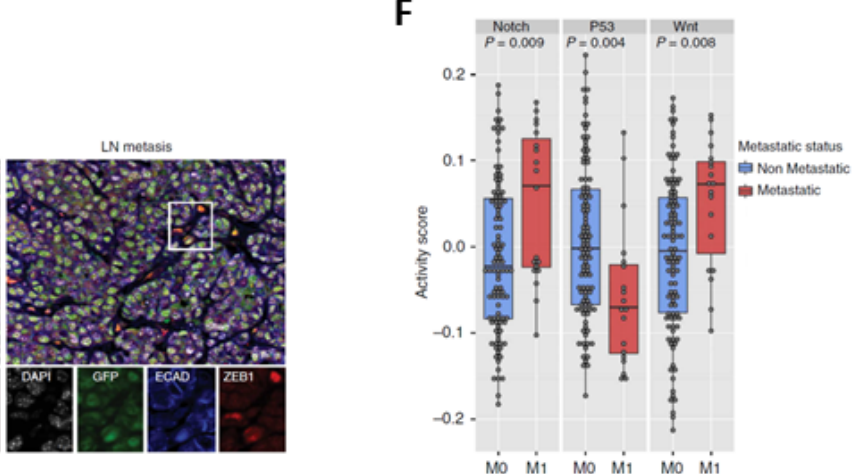

Figure 5. Prediction of synthetic interactions combination to achieve EMT. Mechanistic model of EMT inducers regulation involving Notch (NICD), p53 and Wnt pathways in (A) normal and (B) double mutant with NICD overexpressed and p53 lost. (C) Representation of regulation of three major cell states in colon cancer (cell death, proliferation and metastasis). Lineage tracing by Notch1-GFP and immunofluorescent staining for EMT markers E-Cadherin (ECD), Vimentin and ZEB1 in (D) primary tumor and 
(E) metastases in distant organs; (F) regulation of $\mathrm{p53}$, Notch and Wnt pathways in invasive colon cancer in humans (TCGA data) (Adapted from Chanrion et al., 2014).

\section{Complex stage-specific interventions in the MAPK pathway to disrupt proliferative signaling in bladder cancer}

Structural analysis of biological networks might be useful to find the most optimal SL gene combinations, possibly more than one pair, which might help to remove tumor cells more effectively (Acencio et al., 2013; Huang et al., 2014; Zeng et al., 2014). The MAPK signaling network is coordinated with various processes implicated in cell survival and part of the cell survival map in ACSN (https://acsn.curie.fr/navicell/maps/survival/master/index.html).

The strategy of the following study consists of (i) identification of tumor stage-specific active functional modules, i.e. sets of MAPK signaling network components that are transcriptionally deregulated in bladder cancer (Grieco et al., 2013) compared with controls, and (ii) computation of intervention sets of MAPK map components, whose disruption blocks all the proliferative paths fostered by the identified active functional modules in bladder cancer (Inna Kuperstein et al., 2015a) (Figure 6A). Differential gene expression levels were computed, using transcriptomics data of five different bladder cancer stages. Regions of the map having high density of differentially expressed genes/proteins were identified and scored by GSEA (Gene Set Enrichment Analysis) (Subramanian et al., 2005), being the highly scoring ones assumed to likely indicate sources of proliferative signals in the tumor. If paths exist in the map from the components belonging to any strongly activated network region to the node 'Proliferation', then presumably the region contributes to the activation of cell division (Figure 6A). Using minimal cut sets (computed using the OCSANA algorithm (Vera-Licona et al., 2013a) in the Cytoscape plugin BiNoM (Zinovyev et al., 2008), we removed sets of proteins leading to blockage of the identified proliferative paths. In the less invasive Ta stage, two significantly activated functional modules were identified (Figure 6B; stages Ta). One contains AKT serine/threonine kinase (AKT) from the phosphatidylinositol-4,5-bisphosphate 3-kinase (PI3K) pathway that has been shown to be deeply involved in bladder cancer (Askham et al., 2010). Inhibitors targeting this protein have been recently developed (Ching and Hansel, 2010) and showed promising results. The second module contains the mitogen-activated protein kinase 7 (MAP3K7) protein, an upstream activator of mitogen-activated protein kinase 14 (p38) and mitogen-activated protein kinase 8 (JNK), which is activated by three stimuli: the TNF receptor superfamily member 1 (TNFR1), the Interleukin 1 Receptor Type 1 (IL1R1) and the Transforming Growth Factor Beta Receptor (TGFbR), frequently up-regulated pathways in Ta tumors. OCSANA results suggested that the de-phosphorylation of both p38 and MAP3K7 can block the proliferative effects of MAP3K7- dependent functional module. In the analyzed data-set, the FGFR3 gene was found to be strongly expressed in the stage Ta, less in the stage T1, whereas it has low expression in invasive stages. Strikingly, the best intervention for Ta tumors consists in the disruption of both p38 and a downstream target of PI3K, AKT, that was identified as the most contributing gene with highest differential expression level in the path for the studied dataset (Figure 6B). In the most invasive T2, T3 and T4 stages of bladder cancer, three up-regulated functional modules, with high expression of receptor tyrosine kinase/extracellular signal-regulated kinase (RTK/ERK) signaling components (Figure 6B) were detected. The OCSANA results point to the de-phosphorylation of both ERK and RAS as best interventions to block proliferation, which is consistent with current developments of RAS- and ERK inhibiting drugs for several cancer types (Cakir and Grossman, 2009).

The analysis suggested different interventions depending on the tumor stage. Network analysis using ACSN and OCSANA can be performed for individual patient tumor profiles, leading to personalized treatment recommendations (Grieco, 2013). 
Figure 6

A

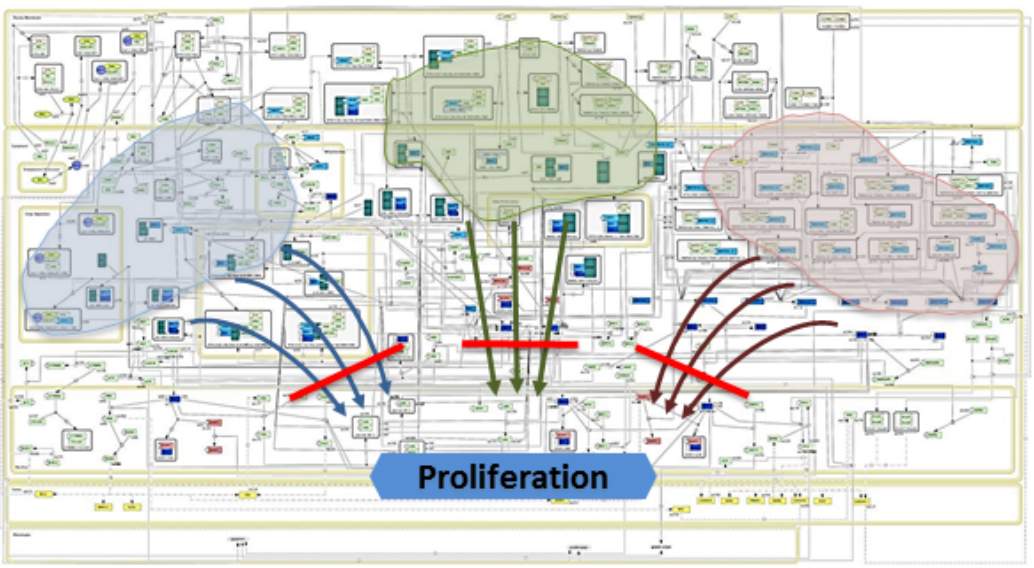

B

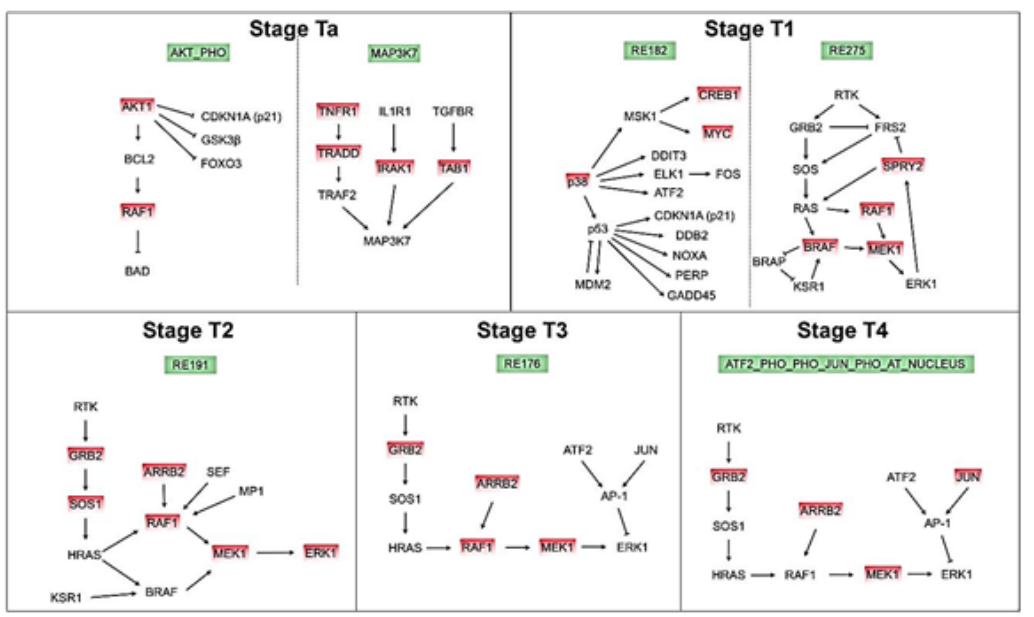

Figure 6. Analytical strategy for finding stage-specific interventions sets using detailed reaction network analysis and omics data. (A) Detailed MAPK network map is shown with schematically indicated activated modules. Finding minimal hitting sets allows cutting all paths (schematically shown by arrows) from the activated modules to the proliferation phenotype. (B) Stage-specific activated modules detected in MAPK network using bladder cancer transcriptome data. Module enrichment score were computed by the GSEA method. The most contributing leading-edge genes with highest differential expression level are highlighted by red. The optimal hitting set lists from these gene elements of MAPK network, which on removal cuts all the paths from the corresponding activated modules to the proliferation phenotype, were calculated using the OCSANA algorithm, and intervention sets for each stage of bladder cancer were suggested. (Adapted from Grieco, 2013).

\section{Finding signaling paths connecting PTP4A3 to metastasis}

PTP4A3 (PRL-3) is able to modulate the key processes that lead to metastasis. This is achieved mainly by targeting major signaling pathways, such as p53, MAPK, mTOR and STAT3, thus promoting proliferation, survival, angiogenesis, motility, etc. In order to understand involvement of PTP4A3 in regulation of specific biological phenotypes that collectively leas to metastasis, the information was first manually retrieved from the scientific literature and systematically gathered together that resulted in a comprehensive signaling map.

In order to identify what paths from PTP4A3 reach and regulate various invasion phenotypes, the OCSANA algorithm in BiNoM (Zinovyev et al., 2008), a Cytoscape plugin, was used. The network path analysis using OCSANA on the PTP4A3 
signaling maps allowed extracting the main paths that lead to various phenotypes that then allow executing the metastasis process. The identified paths in the networks guided further complexity reduction of the network, by maintaining the first order connections, but removing un-related processes from the network. This reduction allows understanding the organization principles of the map and to identify the key paths. In this way, the major players connecting PTP4A3 and the six major phenotypes of interest (cell cycle, survival, angiogenesis, adhesion, cytoskeleton remodeling, EMT and motility and invasion), were retrieved.

PTP4A3 is able to modulate the key processes that lead to metastasis. This is achieved mainly by targeting major signaling pathways, such as p53, MAPK, mTOR and STAT3, thus promoting proliferation and survival. PRL-3 induces angiogenesis via VEGF activation (Figure 7). A strong correlation between PRL-3 and Erk was shown; Erk is well known to allow extracellular matrix adhesion. Even though the role of PTP4A3 on RhoGTPase is not clear (in some studies PTP4A3 activates RhoA and in other the phosphatase down-regulates RhoA expression), PTP4A3 modulates RhoGTPase to promote cytoskeleton remodeling but also EMT and motility, invasion and angiogenesis (Figure 7). The activation of AKT by PTP4A3 is also well established and it promotes survival, EMT and motility, and invasion. Finally, one of the most important players activated by PRL-3 is the kinase Src to promote invasion and metastasis. PTP4A3 also acts by regulating some effectors directly. Indeed, the phosphatase regulates directly integrins, FAK and paxillin involved in focal adhesions or MMPs involved in the degradation of extracellular matrix (Figure 7). PTP4A3 also modulates cytoskeleton proteins such as Ezrin, stathmin and keratin 8 which each of them regulating actin, microtubules and intermediate filaments respectively.

As PTP4A3 is involved in many cancers, this phosphatase appears to be a viable therapeutic target. Some PTP inhibitors are currently used in research but some efforts must be implemented in order to obtain a selective component that can be used in therapy. Worth to notice, these approaches are useful to identify downstream players, which can represent potential targets for further therapeutic strategies. 
Figure 7

A

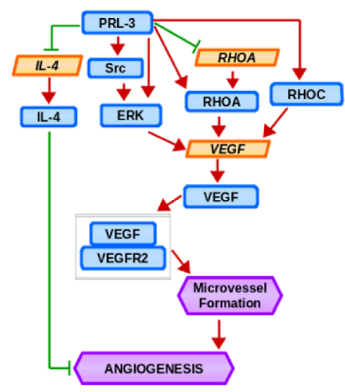

C

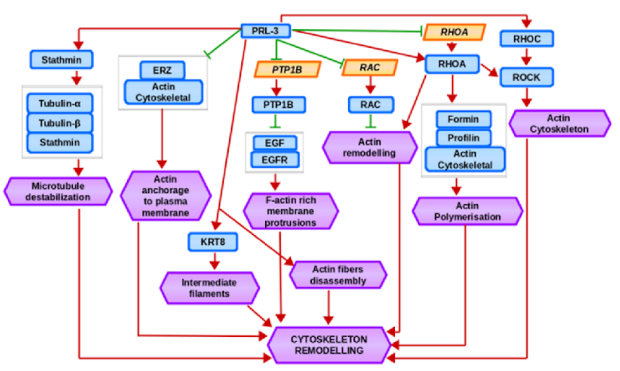

E

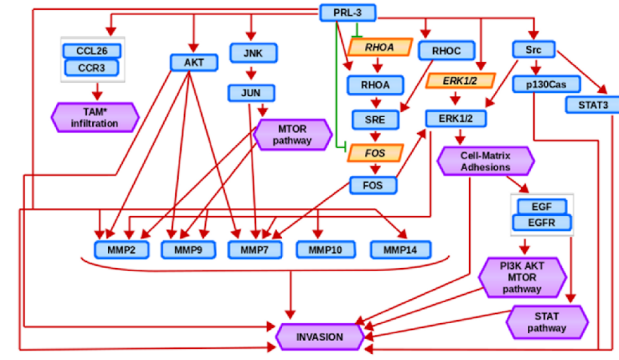

B

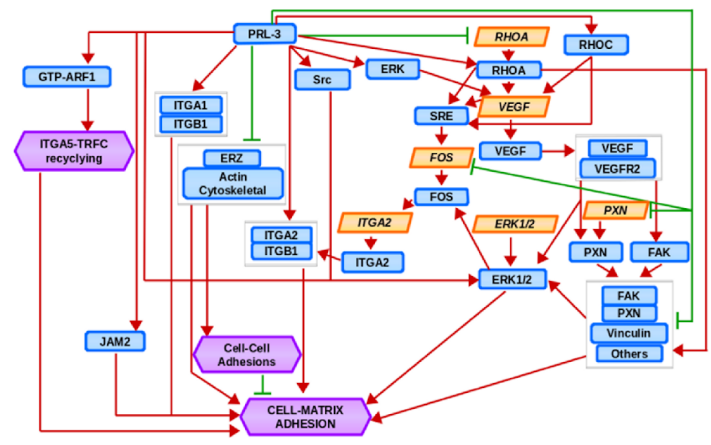

D

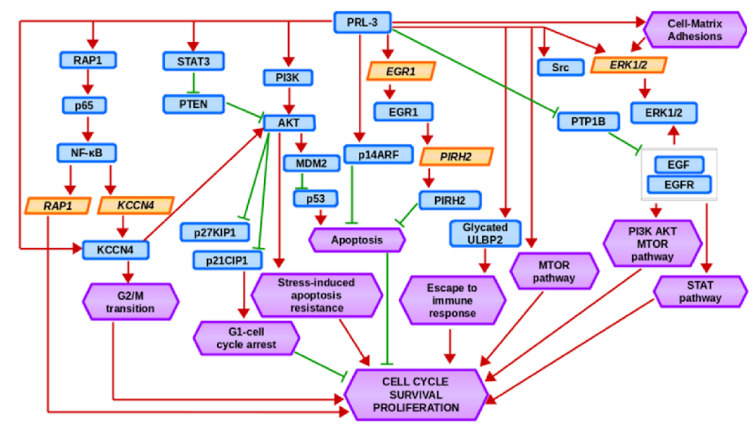

$\mathbf{F}$

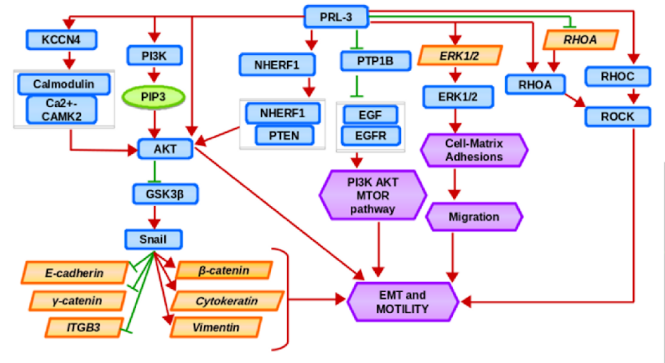

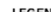

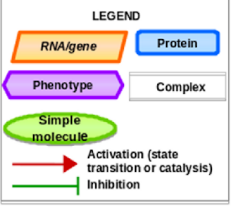

Figure 7. Schematic representations of PTP4A3 (PRL-3) involvement in regulation of the six key phenotypes that collectively contribute to metastasis process in cancer. Interaction diagrams of signaling paths between PTP4A3 to (A) Angiogenesis, (B) Cell Adhesion, (C) Cytoskeleton Remodeling, (D) Cell Cycle, Survival and Proliferation, (E) Invasion and (F) EMT and motility.

\section{Complex intervention gene sets derived from data-driven network analysis for cancer patients resistant to genotoxic treatment}

Genotoxic treatments, like Cisplatin, that induce un-repairable DNA damage and cell death specifically in cancer cells, is often inefficient due to backup mechanisms in the DNA repair signaling. To overcome Cisplatin resistance in ovarian cancer, synthetically interacting combinations of genes can be suggested.

A comprehensive map of cell cycle and DNA repair signaling network constructed from literature curation was used for this study (https://acsn.curie.fr/navicell/maps/dnarepair/master/index.html). The map is composed of three interconnected cell cycle, DNA repair and checkpoints layers covering the most recent knowledge on molecular mechanisms implicated in these processes (I Kuperstein et al., 2015). A state transition graph has been derived from the map including all paths leading to repaired DNA and genes regulating each step (Figure 8A). Using the OCSANA algorithm for searching the minimal cut sets (MCS) on the state transition graphs, considering genes that regulate each step as potential targets for interference (Vera-Licona et al., 2013b). MCSs whose knock-out completely abort DNA 
repair were identified and suggested as potential intervention points (Figure 8B). The coherence of the method has been validated using experimentally-proven SL pairs, verifying the enrichment of the SL pair in real vs. randomlygenerated (pseudo)-MCSs.

For selection of the best MCS to be targeted in patients, one needs to find those MCSs where part of the components is already altered in the patient, allowing exploiting this background and targeting the remaining components in the set, hence achieving synthetic lethality. MCSs were evaluated for each patient (TCGA ovarian cancer dataset) using genomic, expression and mutation data integration and correlation with patient's resistance/sensitivity to Cisplatin. The top-correlated MCSs were ranked according for the mutation status of each gene. Those MCSs that were enriched with genes harboring inactivating mutations were suggested as best intervention combinations to restore sensitivity to Cisplatin by inhibiting the remaining 'active' genes in the set. This approach is relevant for complementing genotoxic chemotherapy by targeting specifically cancer cells and exploiting certain defects in the DNA repair machinery in each patient.

\section{Figure 8}

A

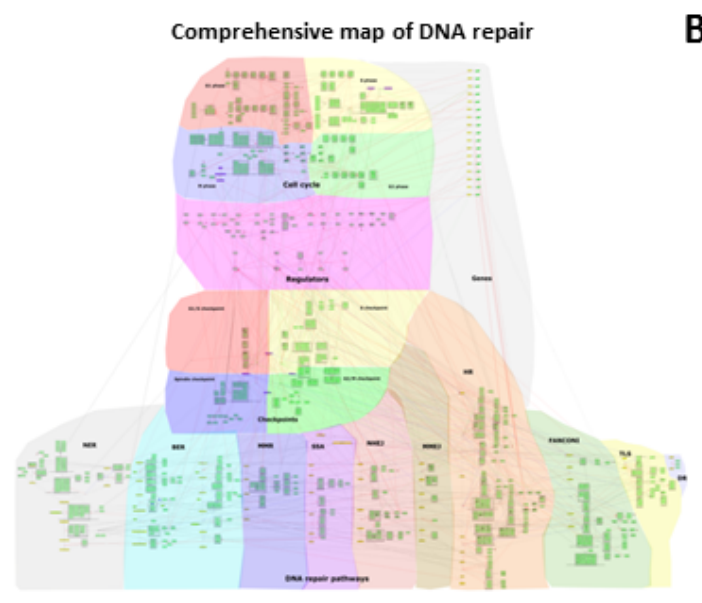

B

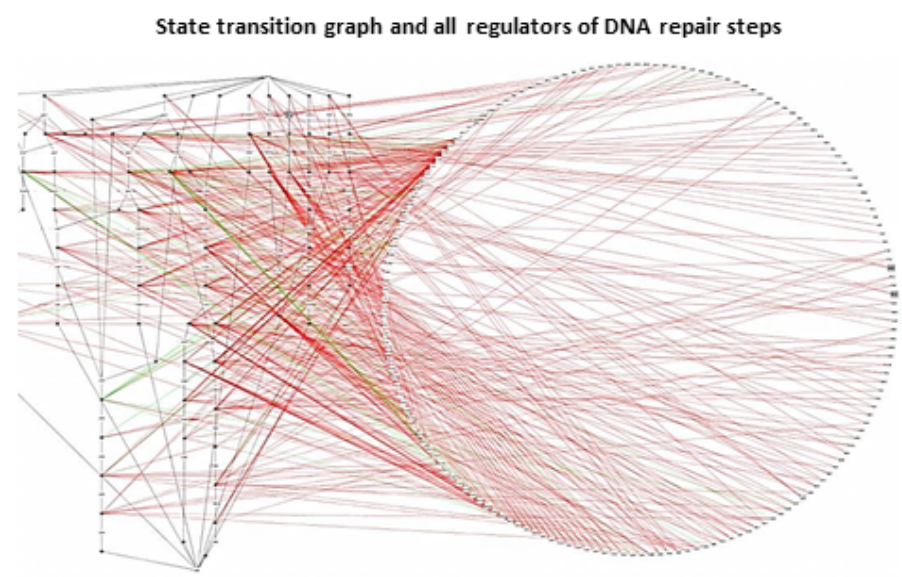

Figure 8. Intervention gene sets for Cisplatin-resistant ovary cancer patients. (A). DNA repair map. (B). State transition graph of DNA repair map with regulators of each state transition (regulators of level 1 ).

\section{ACSN applications in genomic studies}

\section{Finding susceptibility to papillary thyroid carcinoma development}

An example of an association study, where ACSN was used to investigate genes involved in DNA repair, is a case of radiation-induced cancer. Exposure to lonizing radiation is a known cause of cancer, as they produce DNA lesions, and DNA repair genes are at the front line for repairing the effects of those aggressive radiations on the genome. After the Chernobyl nuclear power plant accident in April 1986, an important increase of thyroid malignancies was observed, and in particular of Papillary Thyroid Carcinoma (PTC), in children and adolescent that were exposed to radioactive fallout. We describe an interesting case of genetic association study on papillary thyroid cancer, where cases and controls were both exposed to ionizing radiations after the Chernobyl power plant accident (Lonjou et al., 2017). Belarusian children from the Gomel region (among the most contaminated areas of Belarus), participated to this study: 83 PTC cases and 324 matched and unrelated controls, all being younger than 15 years old at the time of the Chernobyl nuclear accident. 
This study shows that many genes, involved in distinct DNA repair mechanisms, might contribute to the susceptibility to papillary thyroid carcinoma in children exposed to ionizing radiation, as this was the case after the Chernobyl accident. This is a case where both, exposure to ionizing radiation (a known physical carcinogen) and genetic background can result in the development of cancer. Sensitivity to ionizing radiation might be modulated by genetic factors, which could explain inter-individual susceptibility to PTC in exposed individuals. If the children had not been exposed to ionizing radiation, they would not have developed a cancer, so the exposure variable is of primary importance. The genetic factors compound of the disease is (only) a modulating factor. ACSN is a useful resource to study the DNA repair pathway, which is subdivided in 10 modules.

Usually, to gain power in a GWAS study, pooling data from Multi-center studies is done. When pooling data from different origins, in addition to genetic heterogeneity due to different LD patterns, possible heterogeneity in exposure might decrease the power to detect true causal mechanisms in the development of the disease. Ideally, cancer association study design should consider exposure to environmental carcinogens, and always match controls to cases with relevant exposition.

Few genetic studies have both cases and controls exposed to the same carcinogen. In the future, exposome studies, collecting exposure data during all individual's life, will be needed and help refine the importance of combination of genetic background and environmental factors for cancer. Environmental systems epidemiology designed study will be a huge step forward in precision medicine.

\section{Visualization of transcriptome dynamics after inhibition of EWS/FLI-1 in cell lines}

Ewing sarcoma is one of the most frequent forms of pediatric bone tumors. In the majority of patients, a chromosomal translocation induces the expression of the EWS-FLI1 chimeric transcription factor that constitutes the major oncogene in this tumor. The relative genetic simplicity of Ewing sarcoma makes it quite attractive to study cancer in a systematic manner. Silencing EWS-FLI1 leads to cell cycle alteration and, ultimately, results in apoptosis. To study this phenomenon, transcriptome time-series data after EWS-FLI1 silencing were used to identify core modulated genes (Tirode et al., 2007). The transcriptomics data from this experiment depict the temporal changes in gene expression, followed silencing expression of EWS/FLI-1 in mesenchymal stem cells by shRNA. There are in total 10 time points measured during 17 days after EWS/FLI-1 silencing.

Visualization of the cell line transcriptome in the context of the whole ACSN allowed evaluating the global changes in the transcriptome of the tumor cells depending on the status of the EWS-FLI1 (Figure 9). This visualization illustrates the switch of a cancer cell state from tumorigenic and proliferative (Day 0, before inhibiting the EWS/FLI-1 oncogene) to apoptotic and non-proliferative ( Day 9 after inhibition of EWS/FLI-1). When visualizing the data on ACSN, it was observed that genes regulating cell motility had an increased expression (Figure 9). This observation permits to hypothesize that Ewing's sarcoma cells on a less proliferative state are characterized by activated ability to interact with the extracellular matrix and eventually migrate. This hypothesis can be validated by rigorous statistical analysis (Figure 9, C). 
A

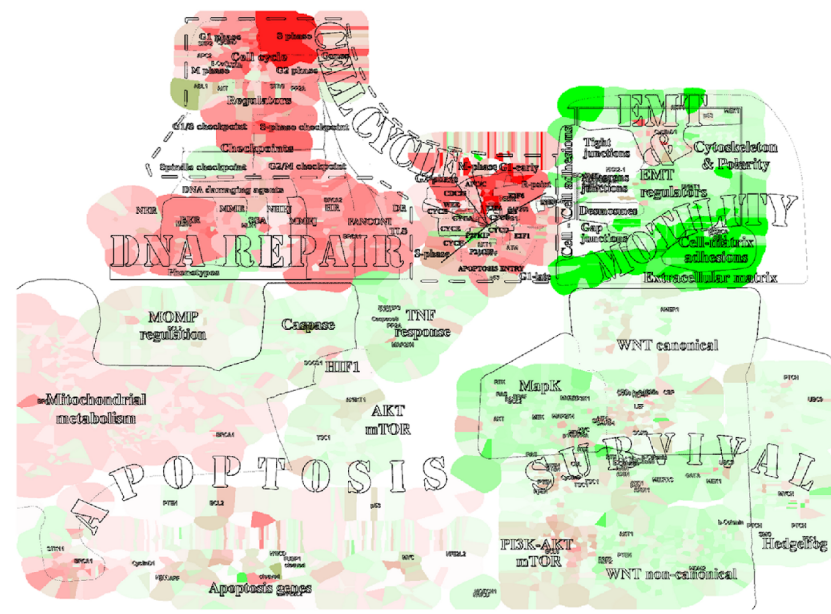

B

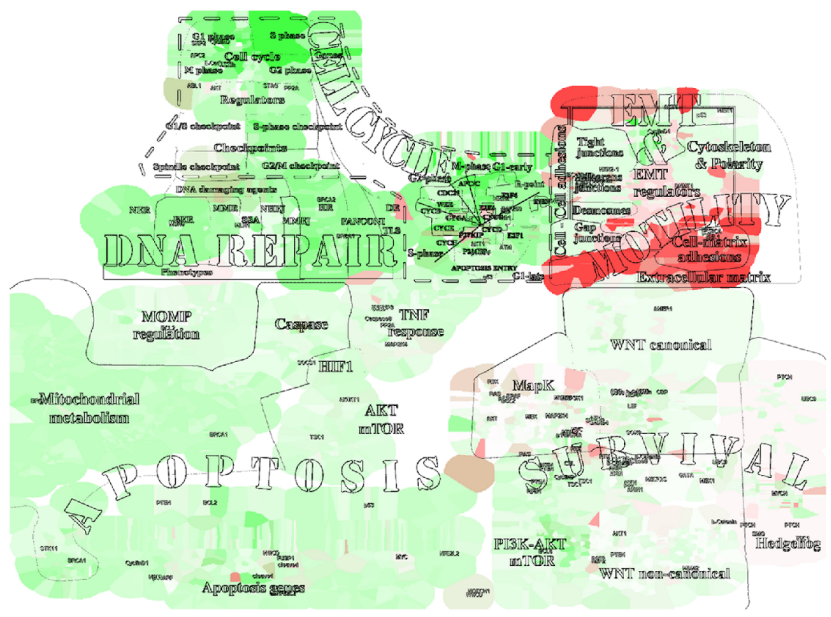

C

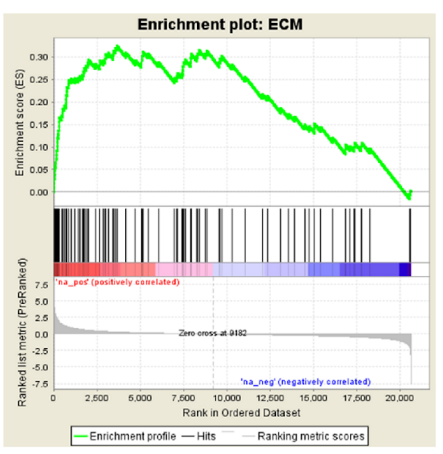

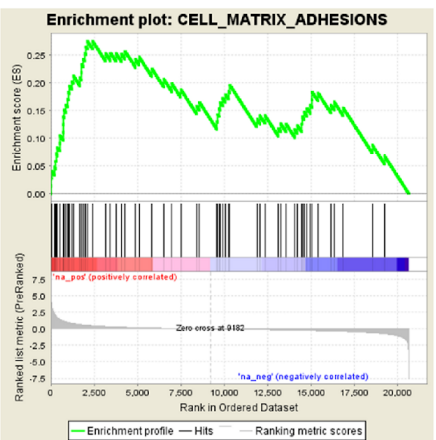
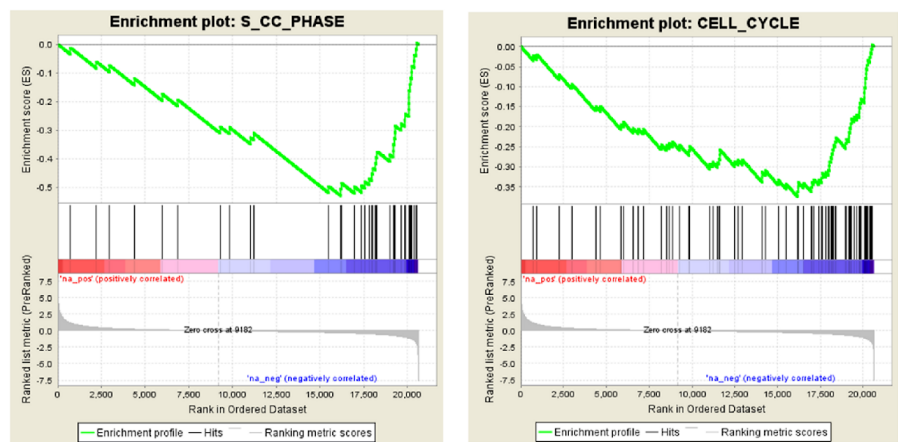

Figure 9. Study of Ewing sarcoma transcriptome dynamics using ACSN. Visualization of cell line transcriptome in the context of the whole ACSN at (A) DAY 0, before inhibiting the EWS/FLI-1 oncogene and (B) DAY9 after inhibition of EWS/FLI-1. The comparison of $A$ and $B$ suggests a switch between proliferative and migrating states of the Ewing's sarcoma cells, visible as drastic change of the color of Cell Cycle and EMT/Cell Motility maps, and especially Cell-matrix adhesion module. (C) GSEA scores calculated using the difference between expression values in Day 9 and Day 0 as and the ACSN modules, indicating significant switch in ACSN modules activation between day 0 and day 9 of the experiment. (Adapted from Bonnet et al., 2015).

\section{Accessibility and downloads}

The ACSN maps can be browsed online via NaviCell platform. The maps components are clickable, making it interactive. The extended annotations of the map components contain a rich tagging system converted to links. This allows tracing the involvement of molecules into different maps and sub-structures as modules. The tagging system also allows to use the map as a source of annotated signatures. The semantic zooming feature of NaviCell simplifies navigation throughout the large collection of molecular interactions, revealing a readable amount of details at each zoom level. A gradual exclusion of details allows the exploration of map contents, going from the detailed towards the top-level view. The hierarchical structure of the ACSN atlas facilitated the generation of several zoom levels for web-based navigation of the map. The ACSN maps can be downloaded from the homepage in several exchange formats as $\mathrm{xml}$, SBGN-ML, BioPax (https://acsn.curie.fr/ACSN2/downloads.html). In addition, the content of the ACSN maps grouped per modules is available and downloadable in the form of GMT files suitable for further functional data analysis. Finally, ACSN2 binary relations between proteins are also available and provided in SIF format. 


\section{Concluding remarks}

In this chapter we demonstrated several examples on ACSN applications for systems medicine. This has been possible thanks to the systematic representations of signaling pathways implicated in the disease, in the form of comprehensive network maps and tools for network-based analysis, visualization and interpretation of cancer omics data.

The aforementioned resource and analytical tools can serve as basis for a more general approach to understand signaling regulation in human disorders, to develop network-based models underlining drug resistance and to suggest intervention sets (Barillot et al., 2013). A workflow that is applicable not only to cancer-related studies, but also to study other pathologies has been depicted.

With the aim of revealing perturbations of molecular mechanisms in different human diseases, to predict drug sensitivity, and to find optimal intervention schemes, one could combine the approaches represented in the examples previously described, into a workflow that contains various steps: (i) construction of a comprehensive intra- and intercellular signaling network of a disease; (ii) integration of omics data and retrieval of network-based signatures, characterizing the disease; (iii) upon data availability, the resistance to treatment can be exploited to elucidate the mechanisms associated with the resistance, being highlighted in a form of deregulated functional modules, pathways or key players; (iv) modelling mechanisms governing the disease and drug resistance and computing intervention gene sets to interfere with the disease and drug resistance. The omics data from each patient can be considered to rank the intervention gene sets according to intrinsic vulnerabilities in each patient.

The suggested approach demonstrated ACSN's wide potential and its applicability for other complex diseases. This rationale has led to the creation of a collective research effort on different human disorders called Disease Maps project (http://disease-maps.org). The Disease Maps community aims at applying similar approaches as described in this review that will lead to identifying emerging disease hallmarks of various disorders, as Parkinson disease (Fujita et al., 2014), influenza (Matsuoka et al., 2013) and others. This will help in studying disease comorbidities; predict response to standard treatments and to suggest improved individual intervention schemes based on drug repositioning (Dorel et al., 2015).

There is an ongoing effort to include ACSN content into various open source platforms and pathway databases, such as (a) MINERVA (Gawron et al., 2016) which is a web-based platform hosting molecular interaction networks encoded as process diagrams in SBGN (Novère et al., 2009) and CellDesigner (Funahashi et al., 2003) formats. MINERVA enables highlighting targets of drugs, and upload and visualization of user-provided experimental datasets, including multiomics entries and custom color coding, (b) Pathway Commons (Cerami et al., 2011) which is a collection of publicly available pathway data from multiple organisms. It provides a web-based interface that enables to browse and search a comprehensive collection of pathways from multiple sources represented in a common language, a download site that provides integrated bulk sets of pathway information in standard or convenient formats and a web service that software developers can use to conveniently query and access all data, (c) WikiPathways (Slenter et al., 2018) which is an open, collaborative platform for capturing and disseminating models of biological pathways for data visualization and analysis. Built on the same MediaWiki software that powers Wikipedia, WikiPathways contains a custom graphical pathway editing tool and integrated databases covering major gene, protein, and small-molecule systems such that it can be used in many projects through standard interfaces as Cytoscape (Shannon et al., 2003), and (d) NDEx (Pratt et al., 2015), the Network Data Exchange, which is an open-source software framework that facilitates the sharing of networks of many types and formats, the publication of networks as data, and the use of networks in modular software. NDEx exists also as a Cytoscape Plugin and can be coupled with various tools available for network analysis or manipulation. 
In addition, by integrating two comprehensive, manually curated network maps such as ACSN and ReconMap 2.0 (Thiele et al., 2013), it will be possible to elucidate the crosstalk between metabolic and signaling pathways. This merge opens an opportunity to model coordination between signaling pathways and metabolism. Multi-level omics data analysis in the context of the signalling and metabolic network maps will allow defining "hot" areas in molecular mechanisms and point to key regulators in physiological or in pathological situations and beyond.

Finally, to make the applications of ACSN broader we ensure that the resource is compatible with, and well connected to downstream analysis pipeline tools. For example, ACSN is part of the GARUDA connectivity platform integrating inter-operable gadgets with applications in biology, healthcare and beyond (Ghosh et al., 2013) (http://www.garudaalliance.org).

\section{References}

Acencio, M.L., Bovolenta, L.A., Camilo, E., Lemke, N., 2013. Prediction of oncogenic interactions and cancer-related signaling networks based on network topology. PLoS One 8, e77521.

Askham, J.M., Platt, F., Chambers, P.A., Snowden, H., Taylor, C.F., Knowles, M.A., 2010. AKT1 mutations in bladder cancer: identification of a novel oncogenic mutation that can co-operate with E17K. Oncogene 29, 150-5.

Avril, T., Vauléon, E., Chevet, E., 2017. Endoplasmic reticulum stress signaling and chemotherapy resistance in solid cancers. Oncogenesis 6, e373.

Barillot, E., Calzone, L., Hupe, P., Vert, J.-P., Zinovyev, A., 2013. Computational Systems Biology of Cancer.

Bonnet, E., Calzone, L., Rovera, D., Stoll, G., Barillot, E., Zinovyev, A., 2013. BiNoM 2.0, a Cytoscape plugin for accessing and analyzing pathways using standard systems biology formats. BMC Syst. Biol. 7, 18.

Bonnet, E., Viara, E., Kuperstein, I., Calzone, L., Cohen, D.P.A., Barillot, E., Zinovyev, A., 2015. NaviCell Web Service for network-based data visualization. Nucleic Acids Res.

Cakir, M., Grossman, A.B., 2009. Targeting MAPK (Ras/ERK) and PI3K/Akt pathways in pituitary tumorigenesis. Expert Opin. Ther. Targets 13, 1121-34.

Cantini, L., Calzone, L., Martignetti, L., Rydenfelt, M., Blüthgen, N., Barillot, E., Zinovyev, A., 2018. Classification of gene signatures for their information value and functional redundancy. npj Syst. Biol. Appl. 4.

Cerami, E., Gao, J., Dogrusoz, U., Gross, B.E., Onur, S., Larsson, E., Antipin, Y., Reva, B., Goldberg, A.P., Sander, C., 2014. The cBio Cancer Genomics Portal: An Open Platform for Exploring Multidimensional Cancer Genomics Data. Cancer Discov. 2, 401-404.

Cerami, E.G., Gross, B.E., Demir, E., Rodchenkov, I., Babur, O., Anwar, N., Schultz, N., Bader, G.D., Sander, C., 2011. Pathway Commons, a web resource for biological pathway data. Nucleic Acids Res. 39, D685-90.

Chanrion, M., Kuperstein, I., Barrière, C., El Marjou, F., Cohen, D., Vignjevic, D., Stimmer, L., Paul-Gilloteaux, P., Bièche, I., Tavares, S.D.R., Boccia, G.-F., Cacheux, W., Meseure, D., Fre, S., Martignetti, L., Legoix-Né, P., Girard, E., Fetler, L., Barillot, E., Louvard, D., Zinovyev, A., Robine, S., 2014. Concomitant Notch activation and p53 deletion trigger epithelial-to-mesenchymal transition and metastasis in mouse gut. Nat. Commun. 5, 5005.

Ching, C.B., Hansel, D.E., 2010. Expanding therapeutic targets in bladder cancer: the PI3K/Akt/mTOR pathway. Lab. Invest. 90, 1406-14.

Chowdhury, S., Sarkar, R.R., 2015. Comparison of human cell signaling pathway databases--evolution, drawbacks and challenges. Database 2015, bau126-bau126.

Costa, A., Kieffer, Y., Scholer-Dahirel, A., Pelon, F., Bourachot, B., Cardon, M., Sirven, P., Magagna, I., Fuhrmann, L., 
Bernard, C., Bonneau, C., Kondratova, M., Kuperstein, I., Zinovyev, A., Givel, A.M., Parrini, M.C., Soumelis, V., VincentSalomon, A., Mechta-Grigoriou, F., 2018. Fibroblast Heterogeneity and Immunosuppressive Environment in Human Breast Cancer. Cancer Cell 33, 463-479.e10.

Czerwinska, U., Calzone, L., Barillot, E., Zinovyev, A., 2015. DeDaL: Cytoscape 3 app for producing and morphing datadriven and structure-driven network layouts. BMC Syst. Biol. 9, 46.

Dorel, M., Barillot, E., Zinovyev, A., Kuperstein, I., 2015. Network-based approaches for drug response prediction and targeted therapy development in cancer. Biochem. Biophys. Res. Commun. 464, 386-91.

Dorel, M., Viara, E., Barillot, E., Zinovyev, A., Kuperstein, I., 2017. NaviCom: a web application to create interactive molecular network portraits using multi-level omics data. Database (Oxford). 2017, 1-11.

Fre, S., Pallavi, S.K., Huyghe, M., Lae, M., Janssen, K.-P., Robine, S., Artavanis-Tsakonas, S., Louvard, D., 2009. Notch and Wnt signals cooperatively control cell proliferation and tumorigenesis in the intestine. Proc. Natl. Acad. Sci. 106, 6309-6314.

Fujita, K.A., Ostaszewski, M., Matsuoka, Y., Ghosh, S., Glaab, E., Trefois, C., Crespo, I., Perumal, T.M., Jurkowski, W., Antony, P.M.A., Diederich, N., Buttini, M., Kodama, A., Satagopam, V.P., Eifes, S., del Sol, A., Schneider, R., Kitano, H., Balling, R., 2014. Integrating Pathways of Parkinson's Disease in a Molecular Interaction Map. Mol. Neurobiol. 49, 88-102.

Funahashi, A., Morohashi, M., Kitano, H. and Tanimura, N. (2003), "CellDesigner: a process diagram editor for generegulatory and biochemical networks", BIOSILICO, available at:https://doi.org/10.1016/s1478-5382(03)02370-9.

Garg, A., Mohanram, K., Di Cara, A., Degueurce, G., Ibberson, M., Dorier, J., Xenarios, I., 2013. Efficient computation of minimal perturbation sets in gene regulatory networks. Front. Physiol. 4, 361.

Gawron, P., Ostaszewski, M., Satagopam, V., Gebel, S., Mazein, A., Kuzma, M., Zorzan, S., McGee, F., Otjacques, B., Balling, R., Schneider, R., 2016. MINERVA-A platform for visualization and curation of molecular interaction networks. npj Syst. Biol. Appl. 2, 1-6.

Ghosh, S., Matsuoka, Y., Asai, Y., Hsin, K.-Y., Kitano, H., 2013. Toward an integrated software platform for systems pharmacology. Biopharm. Drug Dispos. 34, 508-526.

Giampietri, C., Petrungaro, S., Conti, S., Facchiano, A., Filippini, A., Ziparo, E., 2015. Cancer microenvironment and endoplasmic reticulum stress response. Mediators Inflamm. 2015.

Grieco, L., 2013. Integrative modelling and analysis of MAPK network deregulations in human cancers. Aix-Marseille Univ. Fr. 1-51.

Grieco, L., Calzone, L., Bernard-Pierrot, I., Radvanyi, F., Kahn-Perlès, B., Thieffry, D., 2013. Integrative modelling of the influence of MAPK network on cancer cell fate decision. PLoS Comput. Biol. 9, e1003286.

Hanahan, D., Weinberg, R.A., 2011. Hallmarks of cancer: The next generation. Cell.

Huang, L., Li, F., Sheng, J., Xia, X., Ma, J., Zhan, M., Wong, S.T.C., 2014. DrugComboRanker: drug combination discovery based on target network analysis. Bioinformatics 30, i228-36.

Hung, K.E., Maricevich, M.A., Richard, L.G., Chen, W.Y., Richardson, M.P., Kunin, A., Bronson, R.T., Mahmood, U., Kucherlapati, R., 2010. Development of a mouse model for sporadic and metastatic colon tumors and its use in assessing drug treatment. Proc. Natl. Acad. Sci. U. S. A. 107, 1565-70.

Jdey, W., Thierry, S., Russo, C., Devun, F., Al Abo, M., Noguiez-Hellin, P., Sun, J.-S., Barillot, E., Zinovyev, A., Kuperstein, I., Pommier, Y., Dutreix, M., 2017. Drug-Driven Synthetic Lethality: Bypassing Tumor Cell Genetics with a Combination of AsiDNA and PARP Inhibitors. Clin. Cancer Res. 23, 1001-1011. 
Kaushal, V., Dye, R., Pakavathkumar, P., Foveau, B., Flores, J., Hyman, B., Ghetti, B., Koller, B.H., LeBlanc, A.C., 2015. Neuronal NLRP1 inflammasome activation of Caspase-1 coordinately regulates inflammatory interleukin-1-beta production and axonal degeneration-associated Caspase-6 activation. Cell Death Differ. 22, 1676-1686.

Kitano, H., Funahashi, A., Matsuoka, Y., Oda, K., 2005. Using process diagrams for the graphical representation of biological networks. Nat. Biotechnol. 23, 961-966.

Knouf, E.C., Garg, K., Arroyo, J.D., Correa, Y., Sarkar, D., Parkin, R.K., Wurz, K., O’Briant, K.C., Godwin, A.K., Urban, N.D., Ruzzo, W.L., Gentleman, R., Drescher, C.W., Swisher, E.M., Tewari, M., 2012. An integrative genomic approach identifies p73 and p63 as activators of miR-200 microRNA family transcription. Nucleic Acids Res. 40, 499-510.

Kuperstein, I., Bonnet, E., Nguyen, H.-A., Cohen, D., Viara, E., Grieco, L., Fourquet, S., Calzone, L., Russo, C., Kondratova, M., Dutreix, M., Barillot, E., Zinovyev, A., 2015. Atlas of Cancer Signalling Network: a systems biology resource for integrative analysis of cancer data with Google Maps. Oncogenesis 4, e160.

Kuperstein, I., Cohen, D.P.A., Pook, S., Viara, E., Calzone, L., Barillot, E., Zinovyev, A., 2013. NaviCell: A web-based environment for navigation, curation and maintenance of large molecular interaction maps. BMC Syst. Biol. 7, 1.

Kuperstein, I., Grieco, L., Cohen, D.P.A., Thieffry, D., Zinovyev, A., Barillot, E., 2015a. The shortest path is not the one you know: application of biological network resources in precision oncology research. Mutagenesis 30, 191-204.

Kuperstein, I., Robine, S., Zinovyev, A., 2015b. Network biology elucidates metastatic colon cancer mechanisms. Cell Cycle 14, 2189-90.

Lages, J., Shepelyansky, D.L., Zinovyev, A., 2018. Inferring hidden causal relations between pathway members using reduced Google matrix of directed biological networks. PLoS One 13, 1-28.

Liberzon, A., Birger, C., Thorvaldsdóttir, H., Ghandi, M., Mesirov, J.P. and Tamayo, P. (2015), "The Molecular Signatures Database Hallmark Gene Set Collection", Cell Systems, available at:https://doi.org/10.1016/j.cels.2015.12.004.

Lonjou, C., Damiola, F., Moissonnier, M., Durand, G., Malakhova, I., Masyakin, V., Le Calvez-Kelm, F., Cardis, E., Byrnes, G., Kesminiene, A., Lesueur, F., 2017. Investigation of DNA repair-related SNPs underlying susceptibility to papillary thyroid carcinoma reveals MGMT as a novel candidate gene in Belarusian children exposed to radiation. BMC Cancer 17, 328.

Lord, C.J., Tutt, A.N.J., Ashworth, A., 2015. Synthetic lethality and cancer therapy: lessons learned from the development of PARP inhibitors. Annu. Rev. Med. 66, 455-70.

Martignetti, L., Calzone, L., Bonnet, E., Barillot, E., Zinovyev, A., 2016. ROMA: Representation and quantification of module activity from target expression data. Front. Genet. 7, 1-12.

Matsuoka, Y., Matsumae, H., Katoh, M., Eisfeld, A.J., Neumann, G., Hase, T., Ghosh, S., Shoemaker, J.E., Lopes, T.J., Watanabe, T., Watanabe, S., Fukuyama, S., Kitano, H., Kawaoka, Y., 2013. A comprehensive map of the influenza A virus replication cycle. BMC Syst. Biol. 7, 97.

Moes, M., Le Béchec, A., Crespo, I., Laurini, C., Halavatyi, A., Vetter, G., Del Sol, A., Friederich, E., 2012. A novel network integrating a miRNA-203/SNAI1 feedback loop which regulates epithelial to mesenchymal transition. PLoS One 7, e35440.

Musicco, M., Adorni, F., Di Santo, S., Prinelli, F., Pettenati, C., Caltagirone, C., Palmer, K., Russo, A., 2013. Inverse occurrence of cancer and Alzheimer disease: A population-based incidence study. Neurology 81, 322-328.

Nieto, M.A., 2011. The ins and outs of the epithelial to mesenchymal transition in health and disease. Annu. Rev. Cell Dev. Biol. 27, 347-76.

Novère, N. Le, Hucka, M., Mi, H., Moodie, S., Schreiber, F., Sorokin, A., Demir, E., et al. (2009), “The Systems Biology 
Ou, S.M., Lee, Y.J., Hu, Y.W., Liu, C.J., Chen, T.J., Fuh, J.L., Wang, S.J., 2012. Does Alzheimer's disease protect against cancers? A nationwide population-based study. Neuroepidemiology 40, 42-49.

Pratt, D., Chen, J., Welker, D., Rivas, R., Pillich, R., Rynkov, V., Ono, K., et al. (2015), “NDEx, the Network Data Exchange", Cell Systems, available at:https://doi.org/10.1016/j.cels.2015.10.001.

Quanz, M., Berthault, N., Roulin, C., Roy, M., Herbette, A., Agrario, C., Alberti, C., Josserand, V., Coll, J.-L., SastreGarau, X., Cosset, J.-M., Larue, L., Sun, J.-S., Dutreix, M., 2009. Small-molecule drugs mimicking DNA damage: a new strategy for sensitizing tumors to radiotherapy. Clin. Cancer Res. 15, 1308-16.

Salaroglio, I.C., Panada, E., Moiso, E., Buondonno, I., Provero, P., Rubinstein, M., Kopecka, J., Riganti, C., 2017. PERK induces resistance to cell death elicited by endoplasmic reticulum stress and chemotherapy. Mol. Cancer 16, 91.

Sánchez-Valle, J., Tejero, H., Ibáñez, K., Portero, J.L., Krallinger, M., Al-Shahrour, F., Tabarés-Seisdedos, R., Baudot, A., Valencia, A., 2017. A molecular hypothesis to explain direct and inverse co-morbidities between Alzheimer's Disease, Glioblastoma and Lung cancer. Sci. Rep.

Schmid-Burgk, J.L., Chauhan, D., Schmidt, T., Ebert, T.S., Reinhardt, J., Endl, E., Hornung, V., 2016. A genome-wide CRISPR (clustered regularly interspaced short palindromic repeats) screen identifies NEK7 as an essential component of NLRP3 inflammasome activation. J. Biol. Chem. 291, 103-109.

Shannon, P., Markiel, A., Owen Ozier, 2, Baliga, N.S., Wang, J.T., Ramage, D., Amin, N., Schwikowski, B., Ideker, T., 2003. Cytoscape: a software environment for integrated models of biomolecular interaction networks. Genome Res. 2498-2504.

Siemens, H., Jackstadt, R., Hünten, S., Kaller, M., Menssen, A., Götz, U., Hermeking, H., 2011. miR-34 and SNAIL form a double-negative feedback loop to regulate epithelial-mesenchymal transitions. Cell Cycle 10, 4256-71.

Slenter, D.N., Kutmon, M., Hanspers, K., Riutta, A., Windsor, J., Nunes, N., Mélius, J., Cirillo, E., Coort, S.L., Dlgles, D., Ehrhart, F., Giesbertz, P., Kalafati, M., Martens, M., Miller, R., Nishida, K., Rieswijk, L., Waagmeester, A., Eijssen, L.M.T., Evelo, C.T., Pico, A.R., Willighagen, E.L., 2018. WikiPathways: A multifaceted pathway database bridging metabolomics to other omics research. Nucleic Acids Res. 46, D661-D667.

Sperling, R.A., Aisen, P.S., Beckett, L.A., Bennett, D.A., Craft, S., Fagan, A.M., Iwatsubo, T., Jack, C.R., Kaye, J., Montine, T.J., Park, D.C., Reiman, E.M., Rowe, C.C., Siemers, E., Stern, Y., Yaffe, K., Carrillo, M.C., Thies, B., MorrisonBogorad, M., Wagster, M. V., Phelps, C.H., 2011. Toward defining the preclinical stages of Alzheimer's disease: Recommendations from the National Institute on Aging-Alzheimer's Association workgroups on diagnostic guidelines for Alzheimer's disease. Alzheimer's Dement. 7, 280-292.

Subramanian, A., Tamayo, P., Mootha, V.K., Mukherjee, S., Ebert, B.L., Gillette, M.A., Paulovich, A., Pomeroy, S.L., Golub, T.R., Lander, E.S., Mesirov, J.P., 2005. Gene set enrichment analysis: a knowledge-based approach for interpreting genome-wide expression profiles. Proc. Natl. Acad. Sci. U. S. A. 102, 15545-50.

Tabarés-Seisdedos, R., Baudot, A., 2016. Editorial: Direct and Inverse Comorbidities Between Complex Disorders. Front. Physiol. 7, 117.

Thiele, I., Swainston, N., Fleming, R.M.T., Hoppe, A., Sahoo, S., Aurich, M.K., Haraldsdottir, H., et al. (2013), “A community-driven global reconstruction of human metabolism", Nature Biotechnology, available at:https://doi.org/10.1038/nbt.2488.

Tirode, F., Laud-Duval, K., Prieur, A., Delorme, B., Charbord, P., Delattre, O., 2007. Mesenchymal Stem Cell Features of Ewing Tumors. Cancer Cell 11, 421-429.

Trobridge, P., Knoblaugh, S., Washington, M.K., Munoz, N.M., Tsuchiya, K.D., Rojas, A., Song, X., Ulrich, C.M., 
Sasazuki, T., Shirasawa, S., Grady, W.M., 2009. TGF-beta receptor inactivation and mutant Kras induce intestinal neoplasms in mice via a beta-catenin-independent pathway. Gastroenterology 136, 1680-8.e7.

Vera-Licona, P., Bonnet, E., Barillot, E., Zinovyev, A., 2013a. OCSANA: Optimal combinations of interventions from network analysis. Bioinformatics 29, 1571-1573.

Vera-Licona, P., Bonnet, E., Barillot, E., Zinovyev, A., 2013b. OCSANA: optimal combinations of interventions from network analysis. Bioinformatics 29, 1571-3.

Zeng, T., Wang, D.C., Wang, X., Xu, F., Chen, L., 2014. Prediction of dynamical drug sensitivity and resistance by module network rewiring-analysis based on transcriptional profiling. Drug Resist. Updat. 17, 64-76.

Zinovyev, A., Viara, E., Calzone, L., Barillot, E., 2008. BiNoM: a Cytoscape plugin for manipulating and analyzing biological networks. Bioinformatics 24, 876-7.

\section{Relevant websites}

www.acsn.curie.fr Atlas of Cancer Signalling Network, Institut Curie, Paris, France

https://navicell.curie.fr NaviCell web tool, Institut Curie, Paris, France

https://navicom.curie.fr NaviCom web application, Institut Curie, Paris, France

www.cbioportal.org cBioPortal for cancer genomics, Marie-Josée and Henry R. Kravis Center for Molecular Oncology, New York, United States

http://disease-maps.org Disease Maps Project

https://sbgn.github.io/ Systems Biology Graphical Notation project

http://www.celldesigner.org/CellDesigner software, Systems Biology Institute, Tokyo, Japan

https://minerva.pages.uni.lu/doc/ Molecular Interaction Network Visualization Platform, Luxembourg Centre for Systems Biomedicine, Belvaux, Luxembourg

https://www.pathwaycommons.org/ Pathway Commons database, University of Toronto, Toronto, Canada; DanaFarber Cancer Institute and Harvard Medical School, Boston, United States; Oregon Health \& Science University, Oregon, United States.

https://www.ndexbio.org/\#/ NDEx, the Network Data Exchange project, the Cytoscape Consortium; The Regents of the University of California, California, United States

https://www.wikipathways.org/index.php/WikiPathways WIkiPathways database, Maastricht University, Maastricht, the Netherlands; Gladstone Institutes, San Francisco, United States.

https://www.vmh.life/ Virtual Metabolic Human, National University of Ireland, Galway, Ireland.

http://www.garuda-alliance.org Garuda Alliance, Systems Biology Institute, Tokyo, Japan. 


\section{Glossary}

Biocuration: Refers to extraction of biological knowledge from an unstructured text and representation in a formalized computer-readable form.

Biological network: Systems composed of interconnected sub-units. The network is composed of nodes, representing the units or entities, and edges, representing interactions between the nodes.

Cancer Associated Fibroblasts: Are a subset of fibroblasts present in tumors, they show a pathological (activated) phenotype and support tumor growth and metastasis.

Canonical signaling pathways: Is the most common and well-studied variant of a signaling pathway (see signaling pathway).

Exposome: Refers to all the exposures a human encounter through the life-span (diet, drugs, infectious agents, and environmental pollutants, etcetera).

Functional module: Section of signaling map representing a group of molecular interactions with common functional relevance involved in a common process.

Gene-set enrichment analysis (GSEA): Computational approach aimed at finding overrepresented modules in a ranked gene list, using a weighted Kolmogorov-Smirnov test.

Minimal cut set: Minimal set of reactions whose inactivation would lead to a failure in a system function.

Hallmarks of cancer: Are a series of features that help to describe and organize the principles of cancer development.

Invasion: Process of extension and penetration by cancer cells into neighboring tissues.

Metastasis: Process of distant spread of cancer cells from an initial or primary site to a different or secondary site within the host's body.

Pyroptosis: Form of regulated cell death. This process is characterized to be caspase-1 dependent with release of proinflammatory cytokines by the dying cells.

Representation and quantification of module activity (ROMA): Computational method that uses the first component of a principal component analysis to summarize the co-expression of a group of genes in a gene set (module).

Signaling pathway: A cascade of biochemical reactions in the cell that reaches the target molecule or reaction and results in a particular cell phenotype.

Signaling map: Graphical representation of biological knowledge on molecular interactions represented in the form of a diagram of consecutive reactions. 
Tumor Microenvironment: Cellular environment in which the tumor exists, including surrounding blood vessels, immune cells, fibroblasts, bone marrow-derived inflammatory cells, lymphocytes, signaling molecules and the extracellular matrix. Tumor microenvironment includes immune and non-immune cells that play an important role in tumor growth and metastasis. 